\title{
ARTICLE
}

\section{Application of Dose Evaluation of the MCNP Code for the Spent Fuel Transport Cask}

\author{
Mitsufumi ASAMI*, Kenichi SAWADA, Akiko KONNAI and Naoteru ODANO \\ National Maritime Research Institute, 6-38-1, Shinkawa, Mitaka, Tokyo, 181-0004, Japan
}

\begin{abstract}
The spent fuel transport cask has structure based mostly on the multi-layer for the radiation shielding. However, the structure for neutron shielding is very weak around the trunnion. It is important to evaluate realistic dose-equivalent rate in shielding design of the spent fuel transport cask, therefore the three dimensional continuous-energy Monte Carlo radiation transport code that exactly treating the complicated geometry was applied. In the fixed source problem such as a neutron deep penetration calculation with the Monte Carlo method, the application of the variance reduction method is the most important for a high figure of merit and the most reliable calculation. The concerned items are setting method for the variance reduction. The validation of dose-equivalent rate evaluation for the spent fuel transport cask by Monte Carlo code were performed by two kinds of neutron shielding benchmark experiments, deep penetration experiment for the side surface of the cask and streaming experiment around the trunnion. Dose-equivalent rate distributions at each benchmark were measured and compared with the calculated results. The comparison showed a good consistency between calculation and experiment results.
\end{abstract}

KEYWORDS: spent fuel cask, shielding benchmark experiment, streaming, deep penetration, Monte Carlo

\section{Introduction}

Accurately estimating the distribution of radiation dose equivalent rates around a spent fuel shipping cask is fundamental to the analysis for the safety of the cask and the reliability of its shielding design. The radiation shielding structure of the spent fuel transport cask is composed chiefly of the multi-layered structure for the radiation shielding. Neutron shielding may be thin in some parts of the cask such as the periphery of the trunnion, where some neutrons emitted from the source region stream outside of the cask. In spite of the restriction for geometrical expression, the two dimensional discrete ordinates Sn code, DOT3.5, ${ }^{1)}$ has been employed as a typical code for shielding analysis of the cask. Therefore, it is necessary to apply the three dimensional continuous energy Monte Carlo radiation transport code which can treat the complicated geometry of the cask exactly. In the present study, two kinds of neutron shielding benchmark experiment have been performed for the validation of dose-equivalent rate evaluation; deep penetration experiment for the side surface of the cask and streaming experiment for the trunnion. Then radiation shielding calculation was performed and obtained dose-equivalent rate for the comparison with experimental results. The comparison showed a good consistency between calculation and experiment results.

\section{Dose-Equivalent Rate Benchmarks for Spent Fuel Cask \\ 1. Simple Geometry \\ (1) Experimental Model \\ The shielding experiment such as the arrangement mod-}

*Corresponding author, E-mail:asami@nmri.go.jp

(c) 2011 Atomic Energy Society of Japan, All Rights Reserved. eled by the cask cylinder barrel has been conducted in the past. ${ }^{2)}$ In the present study, the effectiveness of the cell importance scheme is examined by the experimental model shown in Fig. 1. Shielding materials are stainless steel, neutron shielding material (NS-4-FR), and lead. ${ }^{252} \mathrm{Cf}$ neutron source is assumed in this examination. Table 1 shows the main specification of the shielding materials. The configuration shown in Table 1 is based on the cylinder barrel of the cask.

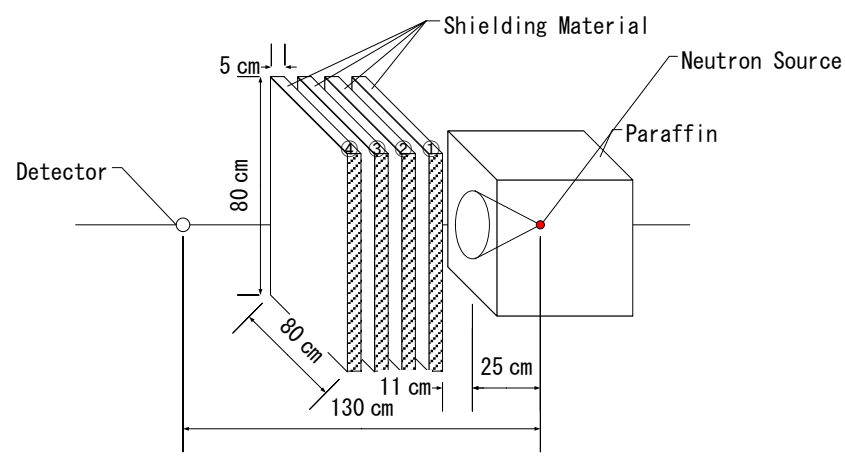

Fig. 1 Schematic arrangement of source, shielding materials and detector.

(2) Monte Carlo Analyses

The measurements were analyzed using 3-D Monte Carlo Code, MCNP. ${ }^{3)}$ MCNP calculations were performed for each thickness of each shielding material, using 100,000,000 histories per case. Variance reduction techniques were applied with the cell importance function. ${ }^{4}$ Nuclear data for interactions with neutrons were taken from the JENDL-3.3. ${ }^{5}$ 
Table 1 The main specification of the shielding material

\begin{tabular}{cll}
\hline \multicolumn{1}{c}{ Configuration } & \multicolumn{1}{c}{ Material } & Thickness \\
\hline & Stainless Steel & $5 \mathrm{~cm}$ \\
& Lead & $10 \mathrm{~cm}$ \\
PWR cask cylinder barrel & Stainless Steel & $5 \mathrm{~cm}$ \\
& NS-4-FR & $15 \mathrm{~cm}$ \\
& Stainless Steel & $5 \mathrm{~cm}$ \\
\hline \multirow{3}{*}{ BWR cask cylinder barrel } & Stainless Steel & $25 \mathrm{~cm}$ \\
& NS-4-FR & $10 \mathrm{~cm}$ \\
& Stainless Steel & $5 \mathrm{~cm}$ \\
\hline
\end{tabular}

The MCMP neutron calculations were performed with the ${ }^{252} \mathrm{Cf}$ fission spectrum defined as following equation.

$$
f(E) \propto \exp [-E / 1.025] \sinh \sqrt{2.926 E}
$$

Table 2 shows the main difference between cell importance and weight window. The weight window variance reduction technique ${ }^{6)}$ adjusts the particle weights as they change phase space, energy and space. In each cell, a lower weight bound and an upper weight bound are specified. If a particle entering a cell has a statistical weight above the upper weight bound, the particle is split such that all split particles are within the weight window. Similarly, if a particle has a weight below the lower weight bound, Russian roulette is used to increase the particle's weight until it lies within the weight window or until it is killed. The weight window variance reduction technique is a space and energy biasing scheme and weight window discriminates on particle weight before taking appropriate action. In order to predict the weight window parameter, it is necessary to know the structure and spectrum of the particle energy in each cell which constitutes the shielding structure. The setup of the unsuitable energy bin on the weight window results in unsuitable splitting and Russian roulette of particles.

Table 2 The main difference between cell importance and weight window

\begin{tabular}{lll}
\hline $\begin{array}{c}\text { Variance reduction } \\
\text { technique }\end{array}$ & Variable dependency & Input value \\
\hline Cell importance & Spatial dependence & Absolute value \\
\hline Weight window & $\begin{array}{l}\text { Spatial and energy } \\
\text { dependence }\end{array}$ & Based on ratios \\
\hline
\end{tabular}

In the cell importance technique, on the other hand, importances are assigned to each cell which makes up the calculation model. Generally, cells near the tally region should have a greater importance than cells standoff. When a particle leaves a cell with importance $I_{1}$ and enters a cell of importance $I_{2}$, the particle is split/rouletted according to the ratio $I_{2} / I_{1}$. For example, if $I_{2} / I_{1}=2.65$, the entering particle is split into 3 particles with $65 \%$ probability and into 2 particles with $35 \%$ probability. If $I_{2} / I_{1}=0.3$, the entering particle is killed with $70 \%$ probability and allowed to survive with $30 \%$ probability. In each splitting or Russian roulette, the weight of the remaining particles is adjusted to leave the tally unbiased. This technique of the cell importance is very reliable unless other biasing techniques are used, and all the particles in a cell will have more or less the same weight regardless of the paths taken to reach the cell. The importance of a cell is intimately related to the average adjoint fluence in the cell. In practice, the cell importances should be adjusted so as to keep the population of particles in the cells relatively constant as one moves from the source region to the tally region. The adjustment of the cell importance is performed by each cell population obtained from the brief calculation. Based on the each cell population, the cell importances are estimated by the ratio of cell populations $P$ in adjacent cells $I_{n} \cong P_{n-1} / P_{n}$. Typically, source cells have an importance of unity and cells closer to the tally region have larger importances. Adjacent cells should not have importances that are significantly different.

Therefore, it is considered that the cell importance is easier to handle the shielding evaluation on the transport cask than the weight window. In the present study, the ratio of cell importances in adjacent cells should not exceed a factor of 4 . The configuration of the cell importance and tally used for the calculation is shown in Table 3.

(3) Calculation Results and Discussions

Figure 2 presents the results of fractional standard deviation (FSD) from the MCNP calculations with the variance reduction, Cell Importance. The FSD of the neutron flux with energy more than $10 \mathrm{MeV}$ becomes large rapidly with the depth of the shielding material and then becomes constant. However, these results do not converge. In the case of the energy less than $10 \mathrm{MeV}$, the FSD of the neutron fluxes becomes large rapidly with the depth of the shielding material, and it became less or equal to reference value $(0.05)$ about FSD of all evaluating points in $x=3.5$ (Here, the reference value is the recommended interpretation of the estimated 1- $\sigma$ confidence interval associated with an MCNP next event estimator tally ${ }^{3)}$ ). Therefore, it is necessary to use a space and energy biasing scheme to evaluate the dose-equivalent rate on the surface of the cask cylinder barrel in the high energy source above $10 \mathrm{MeV}$, take the weight window for example.

\section{Trunnion Region Geometry \\ (1) Experimental Setup and Results}

The trunnions which attach to the spent fuel transport cask are the cylindrical protrusion used as a mounting and pivoting point. In the cargo work, the trunnions are subjected to the cask's own weight. Therefore, the trunnions are directly screwed to the cask body to sustain the load. The region between the trunnion and the cask body has no neutron shielding material: neutrons emitted from the source region in the cask are streamed through the region. In the present study, the trunnion model was made isometrically and the neutron measurements were performed by ${ }^{252} \mathrm{Cf}$ source. The neutron dose-equivalent rates were measured by an ALOKA moderator-type neutron survey meter. Figure 3 shows the trunnion model for the neutron measurement and Fig. 4 shows measurement points around the trunnion.

Table 4 shows the result of the neutron measurements around the trunnion. As expected, neutrons stream from the region without neutron shielding material. 


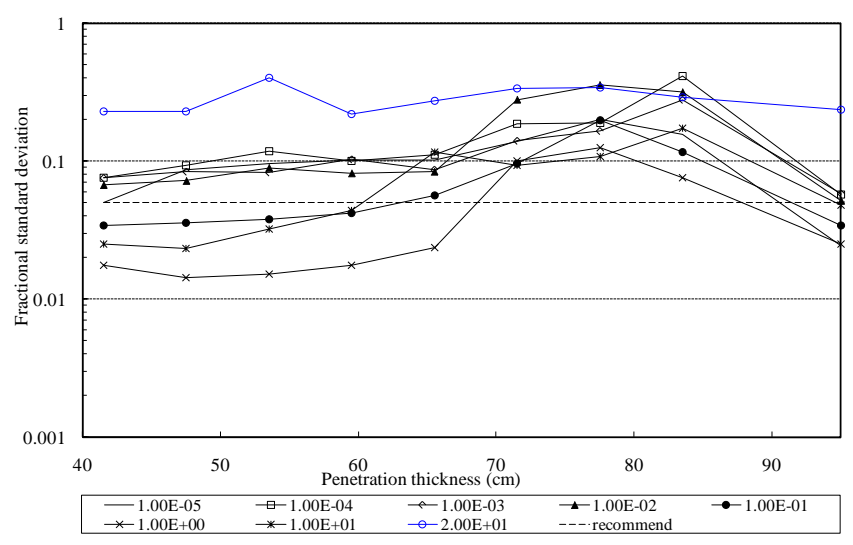

(a) Relation of the depth of the shielding material to the fractional standard deviation with $x=1$. The source energy is considered in the energy region from $1.0 \times 10^{-11} \mathrm{MeV}$ to $20 \mathrm{MeV}$.

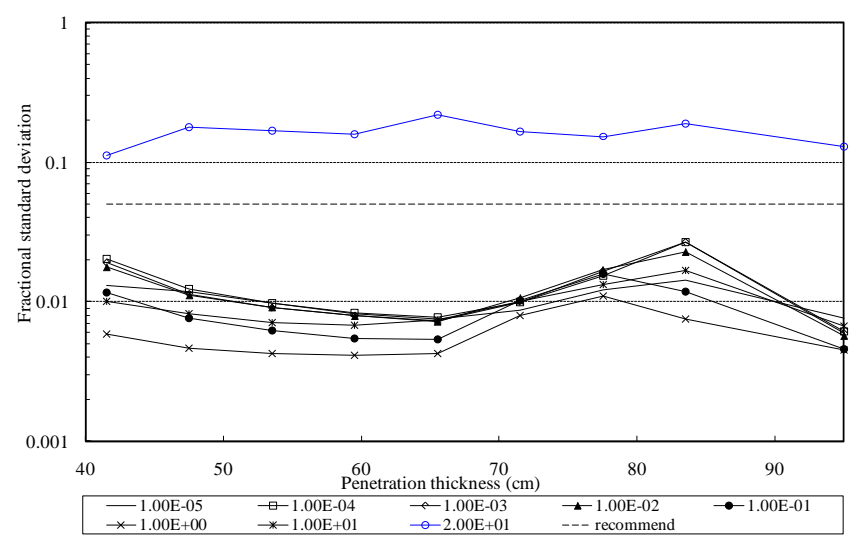

(b) Relation of the depth of the shielding material to the fractional standard deviation with $x=3.5$. The source energy is considered in the energy region from $1.0 \times 10^{-11} \mathrm{MeV}$ to $20 \mathrm{MeV}$.

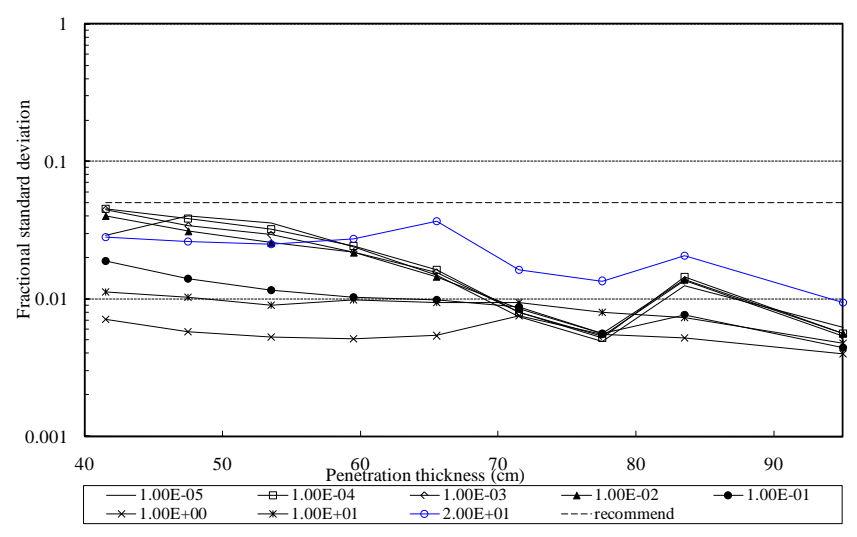

(c) Relation of the depth of the shielding material to the fractional standard deviation with $x=5.0$. The source energy is assumed above $10 \mathrm{MeV}$.

Fig. 2 Relation of the depth of the shielding material to the fractional standard deviation. Each graph legend denotes the energy of the particle $(\mathrm{MeV})$. 'Recommend' denotes the reference value of the standard deviation for the next event estimator tally, 0.05 . Here, the reference value is the recommended interpretation of the estimated 1- $\sigma$ confidence interval associated with an MCNP next event estimator tally. ${ }^{3)}$
Table 3 The configuration of the cell importance and tally used for the calculation

\begin{tabular}{ll}
\hline \multicolumn{1}{c}{ Item } & \multicolumn{1}{c}{ Setting } \\
\hline $\begin{array}{l}\text { The importance change } \\
\text { through overall shielding }\end{array}$ & $10^{x}$ \\
\hline$E \leq 10 \mathrm{MeV}$ & $x=1.0,1.5,2.0,3.5$ \\
\hline$E>10 \mathrm{MeV}$ & $x=5.0,6.0,7.0,8.0,9.0$ \\
\hline Number of histories & 1 Mnps (constant) \\
\hline Thickness of the cell & $\sim$ mean free path \\
\hline Tally & $\begin{array}{l}\text { Next event estimator } \\
\text { (Point detector tally) }\end{array}$ \\
\hline
\end{tabular}

Table 4 Experimental result of the dose equivalent rate around the trunnion. Each column corresponds to the measurement point shown in Fig. 4.

\begin{tabular}{cccccc}
\hline \multicolumn{5}{c}{ Dose-equivalent rate $(\mu \mathrm{Sv} / \mathrm{h})$} \\
\hline \multicolumn{3}{c}{ Trunnion surface } & \multicolumn{2}{c}{$1 \mathrm{~m}$ from the surface } \\
(Statistical error: $\pm 0.1 \mu \mathrm{Sv} / \mathrm{h})$ & (Statistical error: $\pm 0.2 \mu \mathrm{Sv} / \mathrm{h})$ \\
\hline 51.9 & 49.9 & 42.3 & - & - & - \\
\hline 47.7 & 47.2 & 41.9 & 10.8 & 11.0 & - \\
\hline 38.6 & 43.5 & 42.2 & 10.4 & 10.9 & - \\
\hline 38.5 & 42.4 & 41.0 & 10.0 & 10.6 & - \\
\hline 43.3 & 42.5 & 39.4 & - & - & - \\
\hline
\end{tabular}

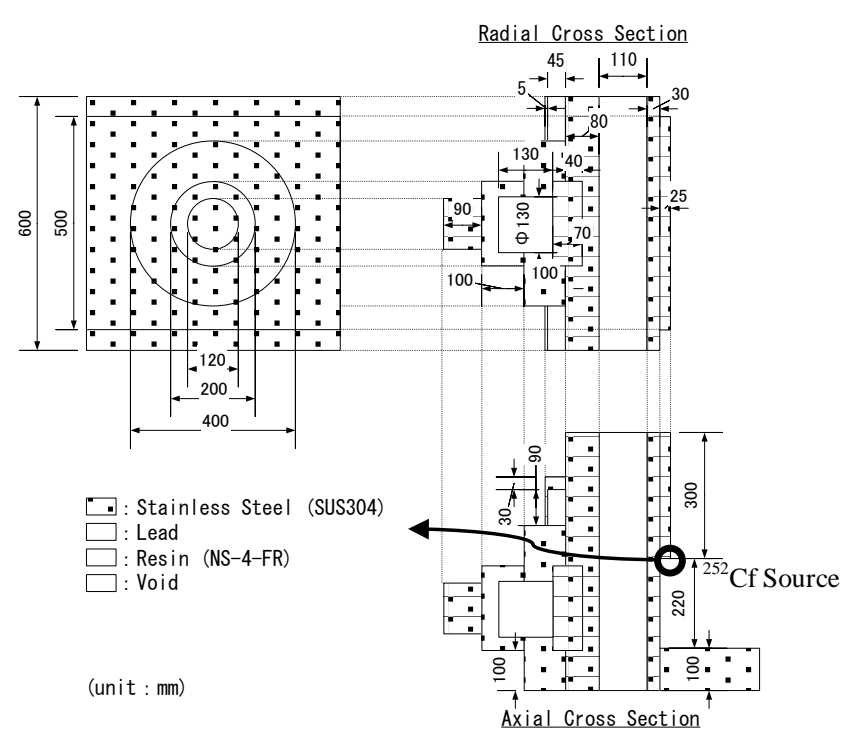

Fig. 3 The trunnion model for the neutron measurement

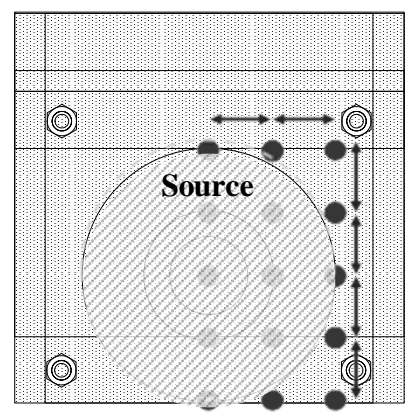

Fig. 4 The measurement points around the trunnion model. All of the length between adjacent points is $10 \mathrm{~cm}$. Shadow area represents the region without neutron shielding material. 
(2) Monte Carlo Analyses

As described in Section II.1.(2), the weight window parameter is determined on the basis of the knowledge of the structure and the spectrum on the particle energy in each cell which constitutes the shielding structure. Without knowledge, the weight window would cause splitting and Russian roulette of particles unsuitably. The weight window genera$\operatorname{tor}^{7)}$ or the adjoint importance function generator ${ }^{8)}$ makes their parameter automatically even though the energy spectrum of the particle is unknown.

In the weight window generator, the weight window parameter is generated by the forward simulation which uses the continuous energy cross section library. Adequate parameter is obtained if the number of particle to reach the estimation point is sufficient. In the case of deep penetration problem, however, it is difficult to get the adequate parameter. To make matters worse, unsuitable weight window parameter is generated by the strong angular dependence of the particle flux.

In the adjoint importance function generator, the weight window parameter is generated by the Monte Carlo adjoint simulation which uses the multi-group cross section library. It is utilized in evaluations where it is more efficient than forward transport calculations, specifically geometries where the detector is relatively small and the source region relatively large. Besides, it generates adjoint importance functions to enhance calculation efficiency in continuous energy Monte Carlo calculations.

In the present study, the adjoint importance function generator, ${ }^{8)}$ which is used for the generation of adjoint importance functions in MCNP, is employed for the variance reduction technique. The cross section library used for the adjoint calculation is MENDF5 ${ }^{8)}$ group constant generated from the code CRSRD. ${ }^{8)}$ To do effective Monte Carlo analysis for the experiments, the superimposed mesh tally ${ }^{3)}$ was used for spatial dose-equivalent rate distribution and the point detector was used for the dose-equivalent rate on measurement point with rem counter. All of the fractional standard deviations in these calculations are within 0.05 . The number of histories for the adjoint calculation was $100,000,000$. The number of histories for the final forward Monte-Carlo simulation, on the other hand, was $100,000,000$.

(3) Calculation Results and Discussions

Figure 5 presents the neutron flux around the trunnion. Figure 5 shows neutrons were streamed from the region without neutron shielding material. It also shows the calculation result of the dose-equivalent rate around the trunnion in Table 5. Calculated-to-experimental ratio $(\mathrm{C} / \mathrm{E})$ of the dose-equivalent rate around the trunnion is shown in Table 6 Table 6 indicates that the calculation is in excellent agreement with the experimental result. In the present study, the calculation with the weight window parameter based on the weight window generator was performed by the same shielding model. The number of histories on the forward calculation for the weight window generator was 1,000,000,000. Table 7 shows the calculation result of the dose-equivalent rate around the trunnion based on the weight
Table 5 Calculation result of the dose-equivalent rate around the trunnion with the weight window parameter on adjoint calculation. Each column corresponds to the measurement point shown in Fig. 4

\begin{tabular}{|c|c|c|c|c|c|}
\hline \multicolumn{6}{|c|}{ Dose-equivalent rate $(\mu \mathrm{Sv} / \mathrm{h})$} \\
\hline \multicolumn{3}{|c|}{$\begin{array}{c}\text { Trunnion surface } \\
\text { (Statistical error: } \pm 0.05 \% \text { ) }\end{array}$} & \multicolumn{3}{|c|}{$\begin{array}{c}1 \mathrm{~m} \text { from the surface } \\
\text { (Statistical error: } \pm 0.05 \% \text { ) }\end{array}$} \\
\hline 52.9 & 51.4 & 47.1 & - & - & - \\
\hline 48.1 & 50.3 & 48.2 & 10.1 & 10.2 & - \\
\hline 37.1 & 44.4 & 47.5 & 10.1 & 10.2 & - \\
\hline 40.2 & 45.3 & 45.5 & 10.1 & 10.2 & - \\
\hline 44.5 & 44.0 & 42.0 & - & - & - \\
\hline
\end{tabular}

Table 6 Calculated-to-experimental ratio (C/E) of the dose-equivalent rate around the trunnion. Each column corresponds to the measurement point shown in Fig. 4.

\begin{tabular}{cccccc}
\hline \multicolumn{7}{c}{ C/E } \\
\hline \multicolumn{3}{c}{ Trunnion surface } & \multicolumn{3}{c}{$1 \mathrm{~m}$ from the surface } \\
\hline 1.02 & 1.03 & 1.11 & - & - & - \\
\hline 1.01 & 1.07 & 1.15 & 0.94 & 0.93 & - \\
\hline 0.96 & 1.02 & 1.13 & 0.97 & 0.94 & - \\
\hline 1.04 & 1.07 & 1.11 & 1.01 & 0.96 & - \\
\hline 1.03 & 1.04 & 1.07 & - & - & - \\
\hline
\end{tabular}

Table 7 Calculation result of the dose-equivalent rate around the trunnion with the weight window parameter on forward calculation. Each column corresponds to the measurement point shown in Fig. 4.

\begin{tabular}{|c|c|c|c|c|c|}
\hline \multicolumn{6}{|c|}{ Dose-equivalent rate (Forward Calc. $\mu \mathrm{Sv} / \mathrm{h}$ ) } \\
\hline \multicolumn{3}{|c|}{$\begin{array}{c}\text { Trunnion surface } \\
\text { (Statistical error: } \pm 0.05 \% \text { ) }\end{array}$} & \multicolumn{3}{|c|}{$\begin{array}{l}1 \mathrm{~m} \text { from the surface } \\
\text { (Statistical error: } \pm 0.05 \%)\end{array}$} \\
\hline 53.0 & 51.5 & 47.1 & - & - & - \\
\hline 48.2 & 50.4 & 48.3 & 10.1 & 10.1 & - \\
\hline 37.2 & 44.5 & 47.5 & 10.1 & 10.1 & - \\
\hline 40.2 & 45.4 & 45.8 & 10.1 & 10.1 & - \\
\hline 44.5 & 43.9 & 41.9 & - & - & - \\
\hline
\end{tabular}

Table 8 Adjoint calculated-to-forward calculated ratio (AC/FC) of the dose-equivalent rate around the trunnion. Each column corresponds to the measurement point shown in Fig. 4.

\begin{tabular}{cccccc}
\hline \multicolumn{6}{c}{ Adjoint calc./Forward calc. } \\
\hline \multicolumn{3}{c}{ Trunnion surface } & \multicolumn{3}{c}{1 m from the surface } \\
\hline 1.00 & 1.00 & 1.00 & - & - & - \\
\hline 1.00 & 1.00 & 1.00 & 1.00 & 1.01 & - \\
\hline 1.00 & 1.00 & 1.00 & 1.00 & 1.01 & - \\
\hline 1.00 & 1.00 & 0.99 & 1.00 & 1.01 & - \\
\hline 1.00 & 1.00 & 1.00 & - & - & - \\
\hline
\end{tabular}

window generator. Adjoint calculated-to-forward calculated ratio (AC/FC) of the dose-equivalent rate around the trunnion is shown in Table 8. Table 8 indicates that both of the calculation results were almost equal except for the history required to obtain converged solutions.

\section{Conclusion}

This study demonstrated the variance reduction for the Monte Carlo shielding calculations of spent fuel transport 


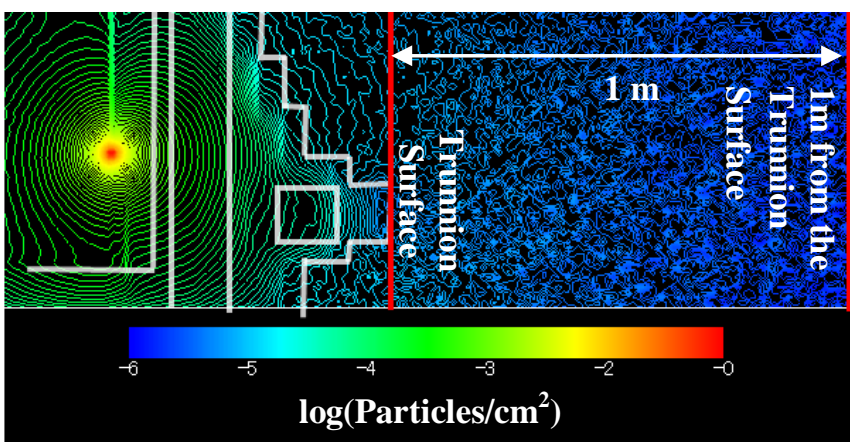

(a) Neutron flux around the trunnion (sectional side view) $\left(\log \left(\right.\right.$ Particles $\left.\left./ \mathrm{cm}^{2}\right)\right)$

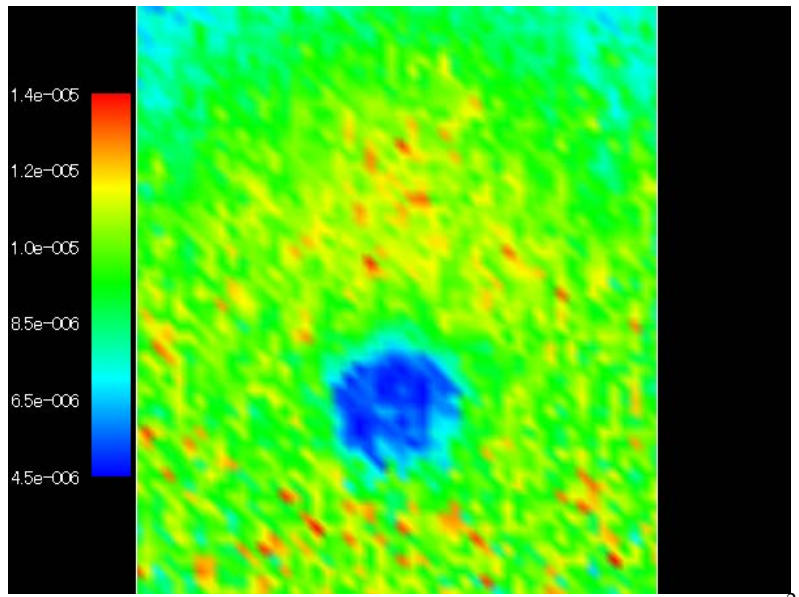

(b) Neutron flux around the surface on the trunnion (Particles $/ \mathrm{cm}^{2}$ )

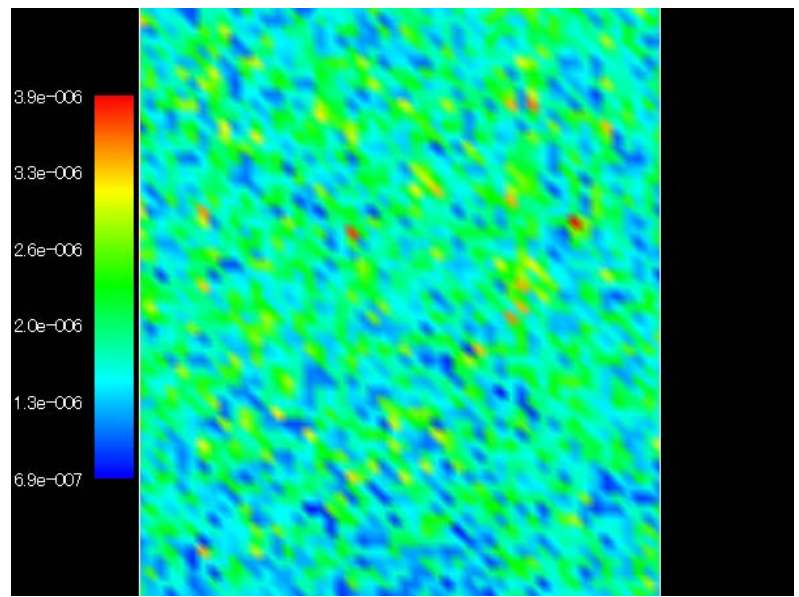

(c) Neutron flux at $1 \mathrm{~m}$ from the surface on the trunnion (Particles/ $\mathrm{cm}^{2}$ )

Fig. 5 The neutron flux around the trunnion. The distribution of neutron flux is obtained by a direct application of the superimposed mesh tally. ${ }^{3)}$ All of the fractional standard deviations in each mesh are within 0.05 . Here, the reference value, 0.05 , is the recommended interpretation of the estimated 1- $\sigma$ confidence interval associated with an MCNP next event estimator tally. ${ }^{3)}$

cask. The radiation shielding structure of the spent fuel transport cask is composed chiefly of the multi-layered structure for the radiation shielding. Some parts of the cask (especially around the trunnion) have thin neutron shielding. For the evaluation by the configuration of the cask cylinder barrel:
By the MCNP calculations with the cell importance, the FSD of the neutron fluxes with energy less than $10 \mathrm{MeV}$ became less or equal to reference value (0.05) about FSD of all evaluating points in $x=3.5$.

The FSD of the neutron flux, which has the energy more than $10 \mathrm{MeV}$, becomes large rapidly with the depth of the shielding material and after that becomes constant, which are not within 0.05 .

- It is necessary to use a space and energy biasing scheme to evaluate the dose-equivalent rate on the surface of the cask cylinder barrel in the high energy source above $10 \mathrm{MeV}$.

For the experiment by the configuration of the trunnion:

- The calculation by the weight window parameter obtained from multi-group adjoint calculation enabled calculation of neutron streaming around the trunnion.

- The calculation by the weight window parameter obtained from weight window generator also enabled calculation of neutron streaming around the trunnion. Number of histories which used for the weight window generator was increased by a factor of 10 compared to multi-group adjoint calculation.

Therefore, multi-group/adjoint option is more useful for calculating the precise shielding calculations than the only spatial biasing scheme (cell importance for example) or the contentious-energy/forward option (the weight window generator for example).

\section{Acknowledgment}

This study was financially supported by the Budget for Nuclear Research of the Ministry of Education, Culture, Sports, Science and Technology, based on the screening and counseling by the Atomic Energy Commission.

\section{References}

1) W. A. Rhodes, F. R. Mynatt, DOT3.5 Two-Dimensional Discrete Ordinates Radiation Transport Code, ORNL-TM-4280 (1973), RSIC Computer Code Collection, CCC-276 (1975).

2) K. Ueki et al., "Neutron Shielding Ability of KRAFTON N2-Mannan KRAFTON N2 Sandwich-type Material and Others," Proc. Topical Mtg. On New Horizons in Radiation Protection and Shielding, ANS, Pasco, (1992).

3) X-5 Monte Carlo Team, MCNP - A General Monte Carlo $N$-Particle Transport Code, Version 5, Los Alamos National Laboratory, LA-UR-03-1987 (2003).

4) J. Spanier, M. Gerbard, Monte Carlo Principles and Neutron Transport Problems, Addison-Wesley Publishing Company (1969).

5) K. Shibata et al., "Japanese Evaluated Nuclear Data Library Version 3 Revision-3: JENDL-3.3,” J. Nucl. Sci. Technol. 39[11], 1125-1136 (2002).

6) T. E. Booth, "Automatic Importance Estimation in Forward Monte Carlo Calculations,” Trans. Am. Nucl. Soc., 41, 308-309 (1982).

7) T. E. Booth, J. S. Hendricks, "Importance Estimation in Forward Monte Carlo Calculations,” Nucl. Technol./Fusion, 5[1], 90-100 (1984).

8) J. C. Wagner et al., MCNP: Multigroup/Adjoint Capabilities, LA-12704 (1994). 